\title{
Effects of Thymoquinone on Adipocyte Differentiation in Human Adipose-Derived Stem Cells
}

Monireh Shahbodi

Mashhad University of Medical Sciences

Seyed Ahmad Emami

Mashhad University of Medical Sciences

Behjat Javadi ( $\sim$ javadib@mums.ac.ir)

Mashhad University of Medical Sciences https://orcid.org/0000-0002-8512-3215

Zahra Tayarani-Najaran

Mashhad University of Medical Sciences

\section{Research Article}

Keywords: Obesity, Fat Stem Cells, Thymoquinone, Black cumin, FAS, PPAR-y

Posted Date: November 30th, 2021

DOI: https://doi.org/10.21203/rs.3.rs-1084907/v1

License: (c) (i) This work is licensed under a Creative Commons Attribution 4.0 International License. Read Full License 


\section{Abstract}

Background: Obesity is one of the most important public health problems worldwide. Stem cells are primary cells capable of differentiating into different types of cells, and can be used to treat various diseases. Thymoquinone (TQ) has antioxidant, anti-inflammatory, anti-diabetic and anti-obesity properties. Herein, we aim to investigate the effect of $T Q$ on the process of lipid differentiation in human adipose tissue-derived stem cells (ADSCs).

Methods and Results: Quantification of cell surface markers was used by Flow-Cytometry and then, the effect of TQ on cell viability was assessed using alamarBlue test. ADSCs were then subjected to induction of differentiation in the presence of non-cytotoxic concentrations of TQ $(6.25,12.5$ and 25 $\mu \mathrm{g} / \mathrm{mL}$ ). ADSCs differentiation was assessed using Oil-Red staining technique. Moreover, expression of $P P A R Y$ (Peroxisome proliferator activated receptor Y) and FAS (Fatty Acid Synthetase) proteins was evaluated using Western blotting analysis. Flow-cytometric analysis demonstrated the expression of CD44 and CD90 markers as mesenchymal stem cells markers on the surface of ADSCs. At concentrations $\leq 100 \mu \mathrm{g} / \mathrm{mL}$ of TQ, no significant difference in cell viability of ADSCs was observed compared to the control. Adipocyte differentiation process significantly decreased at $25 \mu \mathrm{g} / \mathrm{mL}(P<0.001)$ and $12.5 \mu \mathrm{g} / \mathrm{mL}(P<0.01)$ of TQ. The results of the qualitative examination of Lipid Droplets also confirmed these results. Western-blot analysis showed that TQ at $12.5(p<0.05)$ and $25 \mu \mathrm{g} / \mathrm{mL}(p<0.01)$ reduced FAS/ $\beta$-actin ratio compared to the positive group.

Conclusions: This study showed that TQ can reduce the process of differentiation of fat stem cells into fat cells and might be considered as an anti-obesity compound.

\section{Introduction}

Liver and adipose tissue are important organs regulating lipid metabolism in whole body. Adipose tissue is a type of connective tissue containing lipid-rich cells called adipocytes. Obesity is a complex disorder characterized by the excessive lipid accumulation in adipose and liver tissues and may lead to the metabolic syndrome [1]. Recently, obesity has become one of the major health concerns worldwide [2].

The number of adipocytes remains relatively constant after puberty; however, a dramatic increase in adipocyte count (hyperplasia) has been associated with extreme obesity in adults. On the other hand, adipocytes enlargement (hypertrophy) is a common adaptive mechanism to accommodate excessive energy in the form of triglyceride (TG) in adults [1]. It serves to preserve adipose tissue buffering capacity thus protecting other tissues from lipotoxicity [3].

Two types of adipose tissue, "white (WAT)" and "brown (BAT)", are present in human body. WAT is the main site of energy storage, and an endocrine tissue that produces cytokines and adipokines. In obesity condition, WAT is associated with inflammation and oxidative stress leading to insulin resistance and ultimately, metabolic syndrome. BAT is specialized in energy regulation and heat generation through fat burning in response to cold environments and, eventually, to defend against obesity [4] [5]. 
WAT hyperplasia in obesity results in hormonal imbalances and elevated secretion of inflammatory cytokines and adipokines, causing alteration in the normal energy homeostasis which in turn leads to a wide spectrum of diseases. Excessive releases of adipokines can interrupt insulin signaling and cause insulin resistance and eventually type 2 diabetes mellitus (T2DM) [5]. The peroxisome proliferatoractivated receptor- $\gamma$ (PPAR $\gamma$ ) has been considered as a major regulator in adipocyte-differentiation process. Activated PPARy induces the expression of CCAAT-enhancer binding protein a (C/EBPa), which triggers the expression of several genes associated with adipocyte-differentiation to mature adipocyte [6]. On the other hand, fatty acid binding protein 4 (FABP4), adiponectin, and fatty acid synthase (FAS) are key players in the formation of mature adipocytes [5].

Various approaches including dietary regimen, exercise, behavioral modification, and pharmacotherapy are commonly used for lowering body weight. However, these strategies are often ineffective in the long term because they require constant struggle to succeed [7]. Moreover, although conventional anti-obesity medications are to some extent effective; however, the presence of undesirable side effects such as insomnia, constipation, headache, and cardiovascular stroke may limit their acceptability [6]. Therefore, finding and developing anti-obesity drugs with minimum adverse effects would be promising. Today, the use of medicinal plants and traditional medicine systems plays a pivotal role in finding new therapeutic molecules. Natural molecules such as anthocyanins, catechins, and some polyunsaturated fatty acids (PUFA) have shown desirable anti-obesity properties [7]. Nigella sativa L. known as black cumin, is a medicinal plant of Ranunculacea family that has been used in Traditional Persian Medicine (TPM) to attenuate liver problems $[8,9]$ which are important components of metabolic syndrome. N. sativa seed is an important ingredient of several multi-herbal oral and transdermal formulations used to treat diseases caused by cold temperament including obesity and steatohepatitis $[9,10]$. N. sativa seed has been shown to increase both insulin secretion and insulin sensitivity $[11,12]$. Thymoquinone (TQ), a natural quinone $\left(\mathrm{C}_{10} \mathrm{H}_{12} \mathrm{O}_{2}\right)$ abundantly present in $N$. sativa seeds has been reported to possess antiinflammatory, antioxidant, antidiabetic, and anti-hyperlipidemic properties [13, 14]. The antidiabetic effects of TQ is possibly mediated through increasing insulin secretion and maintaining normal carbohydrate metabolism by enhancing glucose utilization and lowering hepatic gluconeogenesis [15] [16].

Adipose-derived stem cells (ADSCs) is considered as the predominant cell type present in the stromal vascular fraction (SVF) of adipose tissue. ADSCs are a group of mesenchymal stem cells with the ability of self-renewal and differentiation into chondrocytes, adipocytes, myocytes, osteoblasts, and even neurocytes [17]. ADSCs have the advantages of being multi-potency, possessing high expansion capacity, being passaged numerously, and cryopreserved for long time [18] and reflecting donor- and depot-specific characteristics [4]. Moreover, ADSCs's harvesting procedure is far less painful than that of bone marrow stem cells [17].

However, to our knowledge, no data on the effect of TQ on cell differentiation and lipid accumulation in ADSCs is available. Therefore, in this study, the effects of TQ on adipocyte differentiation and lipid 
accumulation in human ADSCs based on expression of adipocyte-specific markers FAS and PPARY were investigated.

\section{Materials And Methods \\ 2.1. Materials}

We purchased thymoquinone ( $\geq 99 \%$; CAS No. 490-91-5; CAT No. 274666) from Sigma-Aldrich (Germany), collagenase type1, fetal bovine serum (FBS), penicillin-streptomycin (Pen-Strep) from Gibco (USA), AlamarBlue, Dulbecco's Modified Eagle's Medium (DMEM), Protease inhibitor cocktail, and indomethacin from Sigma (Germany), mouse anti-human CD45-FITC, mouse anti-human CD34-FITC, mouse anti-human CD44-FITC, mouse anti-human CD90-FITC antibodies, $\beta$-actin (8H10D10) Mouse antibody, PPARy (81B8) Rabbit mAb and FAS (C20G5) Rabbit mAb from Cell signaling (USA), Oil Red 0 from Merck (Germany), dexamethasone from Iran hormone (Iran), insulin from Exir (Iran) and ECL Western blot detection reagent from Bio-Rad (USA).

\subsection{Cell culture and differentiation}

Fat samples obtained from liposuction surgery patients aged 38-45 years and body mass index (BMI) $\geq 30 \mathrm{~kg} / \mathrm{m}^{2}$ were transferred to the laboratory in sterile conditions. After washing several times with phosphate buffered saline (PBS) containing $1 \%$ antibiotic, the fat samples were digested by collagenase type $1(0.5 \mathrm{mg} / \mathrm{mL})$ at $37^{\circ} \mathrm{C}$ for $45-60 \mathrm{~min}$. Cell suspension was centrifuged at $1500 \mathrm{rpm}$ for $5 \mathrm{~min}$. The supernatant was discarded and the pellet was re-suspended in Dulbecco's Modified Eagle's Medium (DMEM) supplemented with $10 \% \mathrm{FBS}$, penicillin /streptomycin (Gibco, USA) and incubated at $37^{\circ} \mathrm{C}$ with $5 \% \mathrm{CO}_{2}$. The medium was refreshed twice per week to obtain up to $80 \%$ confluence [19].

\subsection{Quantification of Cell Surface Markers by Flow Cytometry}

Upon third passages, ADSCs were trypsinized and suspended in PBS $\left(10^{\wedge} 6\right.$ cells $\left./ \mathrm{mL}\right)$ and subsequently, were incubated with mouse anti-human CD45-FITC, mouse anti-human CD34-FITC, mouse anti-human CD44-FITC and mouse anti-human CD90-FITC antibodies and incubated for $30 \mathrm{~min}$ at $4^{\circ} \mathrm{C}$ in the dark. PBS was added to the stained cells and centrifuged for $5 \mathrm{~min}$ at $1200 \mathrm{rpm}$. Finally, cells were resuspended in PBS and analyzed by flow cytometry (Becton Dickinson).

\subsection{Cytotoxicity Assay}

ADSCs ( $5 \times 10^{\wedge} 3$ cells/well) were treated with TQ $(6.25,12.5,25,50,100,150$ and $200 \mu \mathrm{g} / \mathrm{mL})$, and incubated with $5 \% \mathrm{CO}_{2}$ at $37{ }^{\circ} \mathrm{C}$ for $24 \mathrm{~h}$. Afterward, $10 \mu \mathrm{l}$ of alamarBlue ${ }^{\circledR}$ was added to each well. The 
intensity of fluorescence was measured at $540 \mathrm{~nm}$ excitation and $560 \mathrm{~nm}$ emission by an ELISA plate reader (Biotech, USA). Each test was repeated three times.

\subsection{Oil Red 0 Staining}

Adipogenesis of human ADSCs was assessed by Oil red O staining. ADSCs from the third passage were cultured in 24-well plates and left to reach $70-80 \%$ density. The cells were then treated with differentiation medium cocktail (DMC) containing $1 \mu \mathrm{M}$ dexamethasone, $10 \mu \mathrm{M}$ insulin, $100 \mu \mathrm{M}$ indomethacin (Sigma, Germany) in DMEM medium (Francis et al. 2010) and maintenance medium cocktail containing $10 \mu \mathrm{M}$ insulin in DMEM medium in the presence or absence of TQ $(6.25,12.5$ and $25 \mu \mathrm{g} / \mathrm{mL})$.

The DMC was replaced every 2 days till day 21 . Then, the cells were fixed with $10 \%$ formaldehyde and stained with Oil Red O. Stained lipids were extracted by isopropanol and the absorbance was measured at $540 \mathrm{~nm}[20]$.

\subsection{Western Blot Analysis}

After 21 days of adipocyte differentiation, the samples were subjected to western blotting analysis to evaluate the expression level of PPARy and, FAS proteins. Then cells were harvested and lysed in cell lysis buffer. $15 \mu \mathrm{L}$ of each sample was loaded onto $10-15 \%$ SDS-polyacrylamide gel and subsequently transferred to polyvinylidene difluoride membranes (Bio-Rad, USA). After blocking in 5\% skim milk dissolved in TBST (TBS plus 0.1\% Tween-20) at room temperature for $2 \mathrm{~h}$, the membranes were incubated with primary antibodies including PPARY (81B8) Rabbit mAb (1:1000) and FAS (C20G5) Rabbit mAb (1:1000)) diluted in TBST (Tris-buffered saline and tween 20). After washing with TBST, the membrane was probed with secondary antibodies (1:3000 in TBST) for $1 \mathrm{~h}$ at $37^{\circ} \mathrm{C}$. $\beta$-actin (8H10D10) Mouse antibody was used as control. The protein bands were detected by Enhanced Chemiluminescent $(E C L)$ Flourchem and analyzed with Image $J$ software. Bands were visualized by chemiluminescent system. Protein expression is normalized against $\beta$-actin level.

\subsection{Statistical Analysis}

All data were presented as Mean \pm SEM. Statistical analysis was performed using Graph Pad Prism 6.0. One-way analysis of variance (ANOVA) were performed for comparing among different groups. $\mathrm{P}<0.05$ was considered to indicate a statistically significant difference.

\section{Results}

\subsection{Characterization of Adipose Derived Stem Cells}


The stem cells isolated from adipose tissue had adhered to the surface of cultured plate during the first days of culturing. The ADSCs rapidly proliferated in vitro and formed fibroblast-like spindle after 5 days of culturing (Fig. 1 a,b,c). Flow cytometric analysis demonstrated the expression of CD44 and CD90 markers as mesenchymal stem cells (MSCs) markers on the surface of ADSCs but were negative for CD45 and CD34 markers (Fig. 1. d).

\subsection{Cytotoxicity Assay}

At concentrations of 100 ( $\mathrm{p} \otimes 0.05$ ), 150 and $200 \mu \mathrm{g} / \mathrm{mL}$ ( $\mathrm{p} \otimes 0.001$ ), TQ decreased the viability of mesenchymal stem cells, but at concentrations below $100 \mu \mathrm{g} / \mathrm{mL}$, no significant difference was observed compared to the control group (Fig. 2).

\subsection{Lipid accumulation}

ADSCs were treated with TQ to investigate its effects on adipocyte differentiation. Adipogenesis process was evaluated using Oil red 0 staining after 21 days. As presented in fig.3, lipid droplets accumulation was detectable as red vacuoles. As results indicated, the amount of Oil-Red 0 stained lipid significantly increased in differentiation group (positive control) compared to control group (untreated with differentiation medium). Adipocyte differentiation process significantly decreased at the concentrations of $25 \mu \mathrm{g} / \mathrm{mL}(P<0.001)$ and $12.5 \mu \mathrm{g} / \mathrm{mL}(P<0.01)$ of TQ. At the concentration of $6.25 \mu \mathrm{g} / \mathrm{mL}$ no significant decrease in differentiation was observed. The results of the qualitative examination of Lipid Droplets also showed that the highest amount of fat vacuoles is formed at the concentration of 6.25 $\mu \mathrm{g} / \mathrm{mL}$ of TQ (Fig.3).

\subsection{Protein Expression Level of PPAR-y and FAS}

Hitherto, the results showed that treatment of ADSCs with TQ decreased lipid accumulation by arresting adipocyte-differentiation. Accordingly, it was hypothesized that the protein expression of key regulators responsible for adipocyte-differentiation, especially FAS and PPARY would be inhibited by TQ. To verify this, we performed Western blot analysis to determine whether TQ suppressed adipocyte differentiation by down-regulating the expression of adipogenic factors, FAS and PPARY. The results of Western blot analysis showed that TQ at $12.5(\mathrm{p}<0.05)$ and $25 \mu \mathrm{g} / \mathrm{mL}(\mathrm{p}<0.01)$ reduced the FAS / $\beta$-actin ratio compared to the positive group. However, at $12.5(\mathrm{p}<0.05)$ and $25 \mu \mathrm{g} / \mathrm{mL}$, TQ had no significant effect on the amount of PPARy protein compared to the positive group while it exhibited a decrease in PPARY protein at $6.25 \mu \mathrm{g} / \mathrm{mL}$ (Fig. 4).

\section{Discussion}


During the past $\sim 50$ years, the prevalence of obesity has increased worldwide, reaching pandemic levels. Obesity can increase the risk of diseases such as T2DM, fatty liver disease, hypertension, cardiovascular problems, dementia, etc., thereby leading to disturbances in quality of life and life expectancy [21]. Adipocyte differentiation plays a key role in the growth of adipose tissue mass and obesity. Thus, inhibition of adipocyte differentiation may be an efficient way to overcome the obesity and related problems. In the present study, the inhibitory effect of TQ on adipocyte differentiation in ADSCs was evaluated.

Nigella sativa seed has been used for centuries to combat various diseases such as diabetes, dyslipidemia and cardiovascular ailments particularly in the Middle East, Southeast Asia and the Mediterranean [22]. Pharmacological studies have revealed its hypoglycemic, anti-hypertensive, antioxidative, anti-hyperlipidemia and antiinflammatory properties. Moreover, $N$. sativa has been shown to moderately reduce body weight, BMI and waist circumference [22-24].

Thymoquinone, the main component of $N$. sativa seeds can improve serum lipid profile by decreasing total lipids, TG and low-density lipoprotein (LDL) levels possibly through diminishing hepatic HMG-CoA reductase activity, enhancing arylesterase activity, regulating genes affecting cholesterol metabolism, and alleviating oxidative stress $[25,26]$. The important characteristic of TQ is the presence of the lipophilic quinine constituent in its structure which efficiently facilitates the entrance of the compound into cellular and subcellular structures, as well as affecting intracellular transcription factors and master regulators [27].

TQ could ameliorate metabolic dysfunction caused by HFD-induced obesity through the activation of genes involved in MPK/PGC1a/SIRT1pathway [28]. TQ also improved glucose tolerance and insulin sensitivity and ameliorated inflammation, lipid dysregulation, and diabetic weight gain in the diet-induced obesity murine model of T2DM possibly via activating SIRT-1 and AMPK pathways [11]. As per the reported studies, we hypostatized that TQ could have beneficial effects in the treatment of obesity. Therefore, we investigated the effects of TQ on adipocyte differentiation and lipid accumulation in an in vitro model of human ADSCs.

In our study, Flow cytometry analysis demonstrated the expression of CD44 and CD90 on the surface of ADSCs as undifferentiated MSCs markers.

TQ at concentrations of $6.25,12.5,25$ and $50 \mu \mathrm{g} / \mathrm{mL}$, exhibited no significant difference in cell viability compared to the untreated control. Numerous studies showed TQ possesses cytotoxic effects in several cancer cell lines such as breast, liver, cervix, brain and colon cancer cell lines in higher concentrations via increasing PPAR-y activity and down-regulating the expression of pro-apoptotic genes including $\mathrm{Bcl}-2$, Bcl$x L$ and survivin $[29,30]$. However, TQ not only has no toxicity in normal cells in low to moderate concentrations but also may protect normal cells against various cytotoxic agents via its antioxidant effect through activation of catalase, superoxide dismutase, glutathione reductase, glutathione-Stransferase and glutathione peroxidase thus preventing lipid peroxidation. Also, TQ could promote the 
expression of HO-1 gene and enhance phosphorylation of Akt and AMPK-a which in turn leads to phosphorylation of Keap1 serine/threonine residues [27].

In the Oil Red $\mathrm{O}$ staining test we observed that adipocyte differentiation process significantly decreased at the concentrations of 25 and $12.5 \mu \mathrm{g} / \mathrm{mL}$ of TQ which is consistent with Shen et al. report in which TQ $(1-4 \mu \mathrm{M})$ could decrease the lipid content and adipocyte expansion in preadipocytes, 3T3-L1 murine fibroblasts [31]. Moreover, TQ downregulated hypoxia-inducible factor 1a (HIF1a) expression in WAT of high-fat diet (HFD)-fed mice. It is known that the expression of HIF1a and secretion of proinflammatory adipokines is triggered by hypoxia induced by adipocyte hypertrophy to mediate obesity-associated insulin resistance [32].

Fatty acid synthase (FAS) is an enzyme which catalyzes the synthesis of long-chain fatty acids in the cytoplasm by condensation of acetyl-CoA and malonyl-CoA, in the presence of NADPH. FAS triggers the formation of mature adipocytes and its inhibition can attenuate preadipocyte differentiation (Fig. 5)[33]. Western blotting analysis revealed that TQ treatment $(12.5$ and $25 \mu \mathrm{g} / \mathrm{mL})$ reduced the FAS / $\beta$-actin ratio compared to the positive group. Gray et al also demonstrated that chronic exposure (72 h) to TQ $(0.5 \mu \mathrm{M})$ decreased the glucose overdose-induced elevation in FAS in rat insulinoma cell line, INS-1 832/13 (a useful model for studying regulation of insulin secretion). Moreover, acute and chronic treatment with TQ and $N$. sativa extract increased glucose-stimulated insulin secretion (GSIS) which was in accordance with their ability to enhance the ATP/ADP ratio. TQ also attenuated the glucose overload-induced GSIS impairment in $\beta$-cells through suppressing the accumulation of malonyl-CoA, increasing acetyl-CoA carboxylase, and fatty acid-binding proteins [34].

PPARY is a ligand-dependent transcription factor that regulates adipocyte differentiation, the expression of genes responsible for lipid metabolism and glucose homeostasis [35]. In the fasting state, PPARa has been shown to accelerate the formation of fatty acid in adipose tissue of the liver through regulating the expression of apolipoprotein, which leads to an increase in plasma levels of high-density lipoprotein cholesterol and a reduction in levels of low-density lipoprotein cholesterol [36]. Activated PPARY decreases free fatty acid content in all organs except adipose tissue and circulating blood, thus causing an increase in the capacity of adipose tissue to store TG [37]. In our study, western blotting revealed that TQ exhibited a decrease in PPARy protein at the low concentration of $6.25 \mu \mathrm{g} / \mathrm{mL}$. This indicates that low concentrations of TQ can suppress PPARy expression leading to a decrease in the capacity of adipose tissue to store TG. To our knowledge, this was the first report on the effects of TQ on PPARy protein expression in ADCs.

In this study, we provided the scientific evidence for the effects of TQ as the main component of $N$. sativa seed which is widely used to manage obesity and metabolic syndrome. The attenuation of adiposity is caused by diminishing both hyperplastic and hypertrophic expansion of

adipocytes [1]. The anti- adipogenesis properties of TQ can be partly attributed to the epigenetic modifications. Recently it was indicated that TQ can modulate epigenetic mechanisms, such as altering histone acetylation and deacetylation, DNA methylation and demethylation, which are epigenetic 
modifications that can contribute to adipogenesis [38]. It was shown that class I histone deacetylases (HDACs; epigenome modifiers that decrease the accessibility for transcription factors to target regions through compacting chromatin) are involved in the adipocytes differentiation and in determining the metabolic characteristics of these cells [39].

\section{Conclusion}

In this study we demonstrated the expression of CD44 and CD90 markers as MSCs markers on the surface of ADSCs by Flow cytometric analysis. At concentrations below $100 \mu \mathrm{g} / \mathrm{mL}$ of $T Q$, there was no significant difference in cell viability of ADSCs compared to the control. Adipocyte differentiation process was significantly reduced at $25 \mu \mathrm{g} / \mathrm{mL}(P<0.001)$ and $12.5 \mu \mathrm{g} / \mathrm{mL}(P<0.01)$ of TQ. The qualitative examination of Lipid Droplets was in harmony with these results. Western blot analysis demonstrated that TQ at $12.5(p<0.05)$ and $25 \mu \mathrm{g} / \mathrm{mL}(p<0.01)$ could decrease the FAS / $\beta$-actin ratio compared to the positive group. Overall, the results of the present study revealed that TQ is capable of inhibiting adipocyte differentiation through reducing FAS and to some extent PPARy proteins expression. Our findings suggest that TQ may serve as a promising natural product for the treatment or prevention of obesity and metabolic disorders in human.

\section{Abbreviations}

PPARY Peroxisome proliferator receptor gamma

GAPDH Glyceraldehyde phosphate dehydrogenase

FAS Fatty Acid Synthetase

TQ Thymoquinone

ADSCs Adipose tissue-derived stem cells

WAT White adipose tissue

BAT Brown adipose tissue

SVF Stromal vascular fraction

PBS Phosphate buffered saline

DMEM Dulbecco's Modified Eagle's Medium

DMC Differentiation medium cocktail

ECL Enhanced Chemiluminescent 
LDL Low-density lipoprotein

HIF1a Hypoxia-inducible factor $1 a$

GSIS Stimulated insulin secretion

\section{Declarations}

\section{Compliance with Ethical Standards}

\section{Funding}

This study was funded by Research Affairs of Mashhad University of Medical Sciences, Mashhad, Iran (Grant No: 950779)

\section{Competing Interests}

The authors have no relevant financial or non-financial interests to disclose

\section{Author's contributions}

All authors contributed to the study conception and design. Material preparation, data collection and analysis were performed by Zahra Tayarani Najaran, Seyed Ahmad Emami, Behjat Javadi and Monireh Shahbodi. The first draft of the manuscript was written by Monireh Shahbodiand Behjat Javadi and all authors commented on previous versions of the manuscript. All authors read and approved the final manuscript.

\section{Ethical approval:}

This article does not contain any studies with human participants or animals performed by any of the authors.

\section{References}

1. Okla M, Kang I, Kim DM, Gourineni V, Shay N, Gu L, Chung S (2015) Ellagic acid modulates lipid accumulation in primary human adipocytes and human hepatoma Huh7 cells via discrete mechanisms. J Nutr Biochem. 26: 82-90

2. Li Y, Rong Y, Bao L, Nie B, Ren G, Zheng C, Amin R, Arnold RD, Jeganathan RB, Huggins KW (2017) Suppression of adipocyte differentiation and lipid accumulation by stearidonic acid (SDA) in 3T3-L1 
cells. Lipids Health Dis 16:1-10

3. Muir LA, Neeley CK, Meyer KA, Baker NA, Brosius AM, Washabaugh AR, Varban OA, Finks JF, Zamarron BF, Flesher CG (2016) Adipose tissue fibrosis, hypertrophy, and hyperplasia: Correlations with diabetes in human obesity. Obesity 24:597-605

4. Ruiz-Ojeda FJ, Rupérez Al, Gomez-Llorente C, Gil A, Aguilera CM (2016) Cell models and their application for studying adipogenic differentiation in relation to obesity: a review. Int J Mol Sci 17:1040

5. Moseti D, Regassa A, Kim W-K (2016) Molecular regulation of adipogenesis and potential antiadipogenic bioactive molecules. Int J Mol Sci 17:124

6. Sugimoto R, Ishibashi-Ohgo N, Atsuji K, Miwa Y, Iwata O, Nakashima A, Suzuki KEuglena extract suppresses adipocyte-differentiation in human adipose-derived stem cells. (2018) PloS one.13:e0192404

7. Park M, Sharma A, Lee H-J (2019) Anti-adipogenic effects of delphinidin-3-O- $\beta$-glucoside in 3T3-L 1 preadipocytes and primary white adipocytes. Molecules. 24: 1848

8. Aqili Khorasani MH, Qarabadin- (2007) e-Kabir. Tehran: Research Institute for Islamic and Complementary Medicine (RICM)

9. Razi MZ (1968) Al-Hawi fi'l-Tibb (Comprehensive Book of Medicine). Osmania Oriental Publications Bureau, Hyderabad

10. Razavi B, Hosseinzadeh H (2014) A review of the effects of Nigella sativa L. and its constituent, thymoquinone, in metabolic syndrome. J Endocrinol Invest 37:1031-1040

11. Karandrea S, Yin H, Liang X, Slitt AL, Heart EA (2017) Thymoquinone ameliorates diabetic phenotype in Diet-Induced Obesity mice via activation of SIRT-1-dependent pathways. PloS one. 12: e0185374

12. Mohebbati R, Abbasnezhad A (2020) Effects of Nigella sativa on endothelial dysfunction in diabetes mellitus: A review. J Ethnopharmacol 252:112585

13. Parlar A, Arslan SO (2019) Thymoquinone exhibits anti-inflammatory, antioxidant, and immunomodulatory effects on allergic airway inflammation. Archives of Clinical Experimental Medicine. 4: 60-65

14. Pei Z-W, Guo Y, Zhu H-L, Dong M, Zhang Q, Wang F (2020) Thymoquinone Protects against Hyperlipidemia-Induced Cardiac Damage in Low-Density Lipoprotein Receptor-Deficient (LDL-R-/-) Mice via Its Anti-inflammatory and Antipyroptotic Effects. Biomed Res Int. 2020

15. Fararh K, Shimizu Y, Shiina T, Nikami H, Ghanem M, Takewaki T (2005) Thymoquinone reduces hepatic glucose production in diabetic hamsters. Vet Sci Res J 79:219-223

16. Pari L, Sankaranarayanan C (2009) Beneficial effects of thymoquinone on hepatic key enzymes in streptozotocin-nicotinamide induced diabetic rats. Life Sci 85:830-834

17. Konno M, Hamabe A, Hasegawa S, Ogawa H, Fukusumi T, Nishikawa S, Ohta K, Kano Y, Ozaki M, Noguchi Y Adipose-derived mesenchymal stem cells and regenerative medicine. (2013) Dev Growth Differ.55:309-318 
18. Lee M-J, Fried SOptimal protocol for the differentiation and metabolic analysis of human adipose stromal cells. (2014) Meth Enzymol. 538:49-65

19. Wilson A, Chee M, Butler P, Boyd AS (2019) Isolation and characterisation of human adipose-derived stem cells, in Immunological tolerance. Springer, pp 3-13

20. Ong WK, Tan CS, Chan KL, Goesantoso GG, Chan XHD, Chan E, Yin J, Yeo CR, Khoo CM, So JBY Identification of specific cell-surface markers of adipose-derived stem cells from subcutaneous and visceral fat depots. (2014) Stem Cell Rep. 2:171-179

21. Blüher M (2019) Obesity: global epidemiology and pathogenesis. Nat Rev Endocrinol 15:288-298

22. Namazi N, Larijani B, Ayati MH, Abdollahi M (2018) The effects of Nigella sativa L. on obesity: A systematic review and meta-analysis. J ethnopharmacol 219:173-181

23. Asgary S, Ghannadi A, Dashti G, Helalat A, Sahebkar A, Najafi S (2013) Nigella sativa L. improves lipid profile and prevents atherosclerosis: Evidence from an experimental study on hypercholesterolemic rabbits. J Funct Foods 5:228-234

24. Mahmoodi MR, Mohammadizadeh M (2020) Therapeutic potentials of Nigella sativa preparations and its constituents in the management of diabetes and its complications in experimental animals and patients with diabetes mellitus: A systematic review.Complement Ther Med:102391

25. Ahmad S, Beg ZH (2013) Hypolipidemic and antioxidant activities of thymoquinone and limonene in atherogenic suspension fed rats. Food chem. 138: 1116-1124

26. AL-NAQEEB G, Ismail M (2009) Regulation of apolipoprotein A-1 and apolipoprotein B100 genes by thymoquinone rich fraction and thymoquinone in HepG2 cells. J Food Lipids 16:245-258

27. Mahmoud YK, Abdelrazek HM (2019) Cancer: Thymoquinone antioxidant/pro-oxidant effect as potential anticancer remedy. Biomed Pharmacother. 115: 108783

28. Harphoush S, Wu G, Qiuli G, Zaitoun M, Ghanem M, Shi Y, Le G (2019) Thymoquinone ameliorates obesity-induced metabolic dysfunction, improves reproductive efficiency exhibiting a dose-organ relationship. Syst Biol Reprod Med 65:367-382

29. Imran M, Rauf A, Khan IA, Shahbaz M, Qaisrani TB, Fatmawati S, Abu-Izneid T, Imran A, Rahman KU, Gondal TA (2018) Thymoquinone: A novel strategy to combat cancer: A review. Biomed Pharmacother. 106: 390-402

30. Darakhshan S, Pour AB, Colagar AH, Sisakhtnezhad SThymoquinone and its therapeutic potentials. (2015) Pharmacol Res.95:138-158

31. Shen HH, Peterson SJ, Bellner L, Choudhary A, Levy L, Gancz L, Sasson A, Trainer J, Rezzani R, Resnick A (2020) Cold-Pressed Nigella Sativa Oil Standardized to 3\% Thymoquinone Potentiates Omega-3 Protection against Obesity-Induced Oxidative Stress, Inflammation, and Markers of Insulin Resistance Accompanied with Conversion of White to Beige Fat in Mice. Antioxidants. 9: 489

32. Chen $\mathrm{L}$, Chen $\mathrm{R}$, Wang $\mathrm{H}$, Liang $\mathrm{F}$ (2015) Mechanisms linking inflammation to insulin resistance. (2015) Int J Endocrinol. 
33. Ronnett GV, Kim E-K, Landree LE, Tu Y (2005) Fatty acid metabolism as a target for obesity treatment. Physiol Behav 85:25-35

34. Gray JP, Burgos DZ, Yuan T, Seeram N, Rebar R, Follmer R, Heart EA Thymoquinone, a bioactive component of Nigella sativa, normalizes insulin secretion from pancreatic $\beta$-cells under glucose overload via regulation of malonyl-CoA. (2016) Am J Physiol Endocrinol Metab. 310: E394-E404

35. Lee JM, Choi SS, Lee YH, Khim KW, Yoon S, Kim B-g, Nam D, Suh P-G, Myung K, Choi JH (2018) The E3 ubiquitin ligase TRIM25 regulates adipocyte differentiation via proteasome-mediated degradation of PPARy. Exp Mol Med 50:1-11

36. Hong F, Pan S, Guo Y, Xu P, Zhai Y (2019) PPARs as nuclear receptors for nutrient and energy metabolism. Molecules. 24: 2545

37. Gross B, Pawlak M, Lefebvre P, Staels B (2017) PPARs in obesity-induced T2DM, dyslipidaemia and NAFLD. Nat Rev Endocrinol 13:36

38. Khan MA, Tania M, Fu J (2019) Epigenetic role of thymoquinone: impact on cellular mechanism and cancer therapeutics. Drug Discov Today 24:2315-2322

39. Ferrari A, Longo R, Peri C, Coppi L, Caruso D, Mai A, Mitro N, De Fabiani E, Crestani M (2020) Inhibition of class I HDACs imprints adipogenesis toward oxidative and brown-like phenotype. Biochim Biophys Acta Mol Cell Biol Lipids 1865:158594

\section{Figures}




\section{Fig. 1}
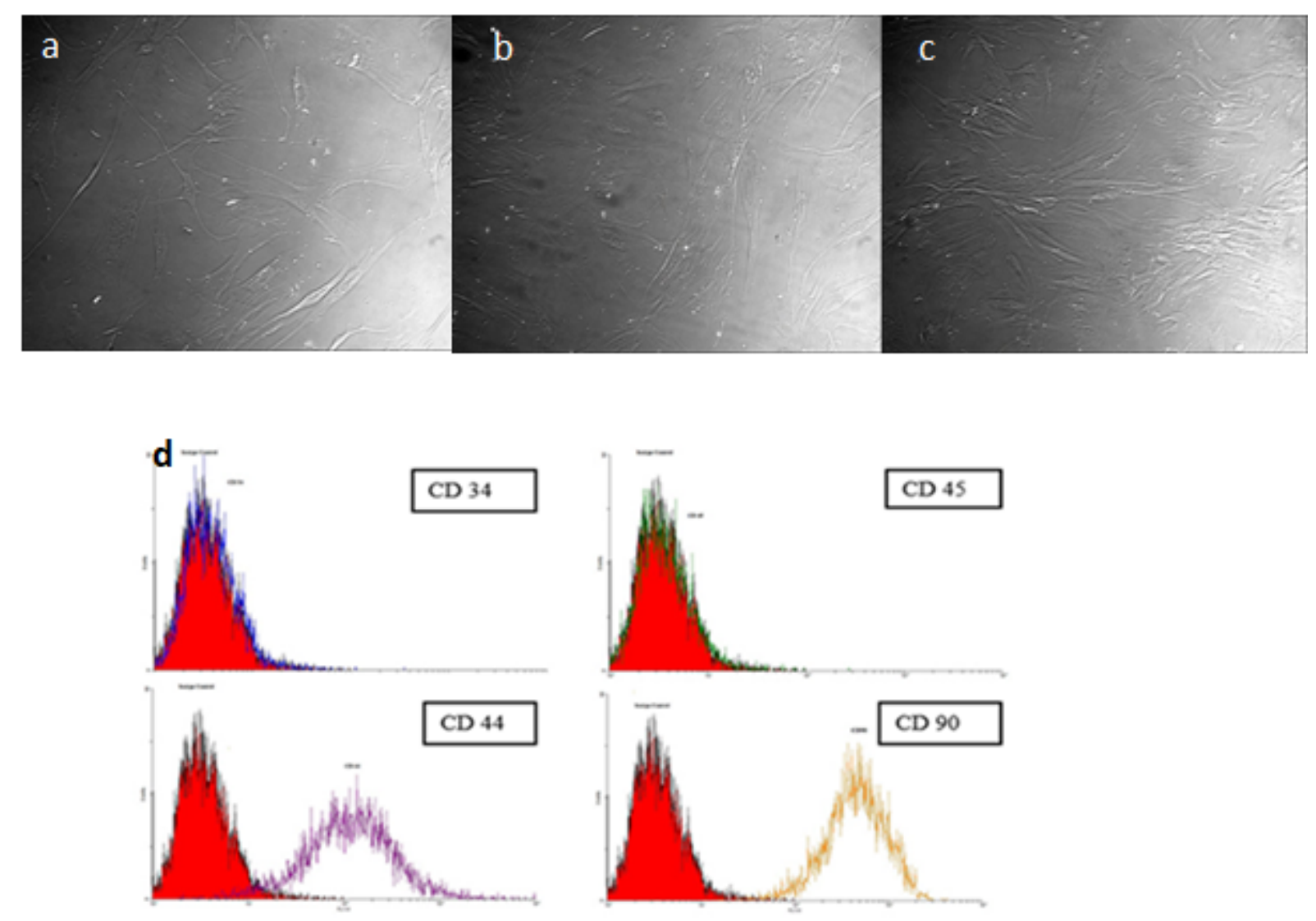

\section{Figure 1}

ADSCs under inverted microscope (10x magnification) and Flow cytometric analysis of ADSCs a: ADSCs 3 days after isolation. b: ADSCs 5 days after isolation. c: ADSCs 10 days after isolation. d: Flow cytometric analysis of ADSCs was demonstrated the positive expression of CD90 
Fig. 2

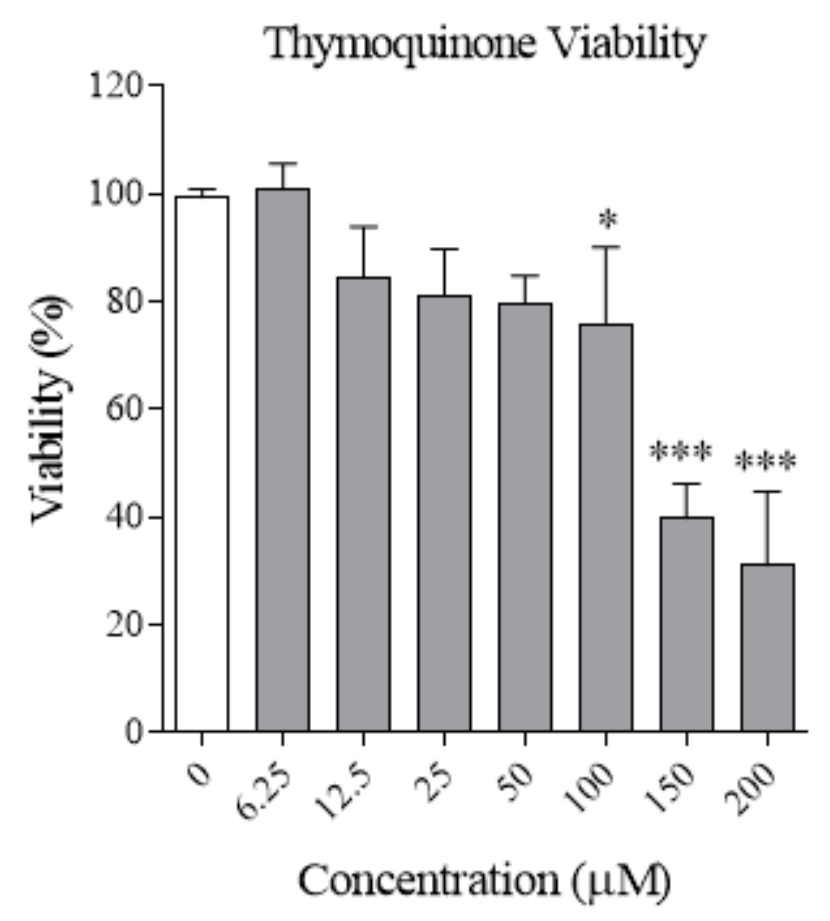

Figure 2

Cell viability of ADSCs after treatment with different concentrations of TQ for $24 \mathrm{~h}$. m. Data are represented as means $\pm S D(n=3) .{ }^{*}=p<0.05$ 


\section{Fig. 3}

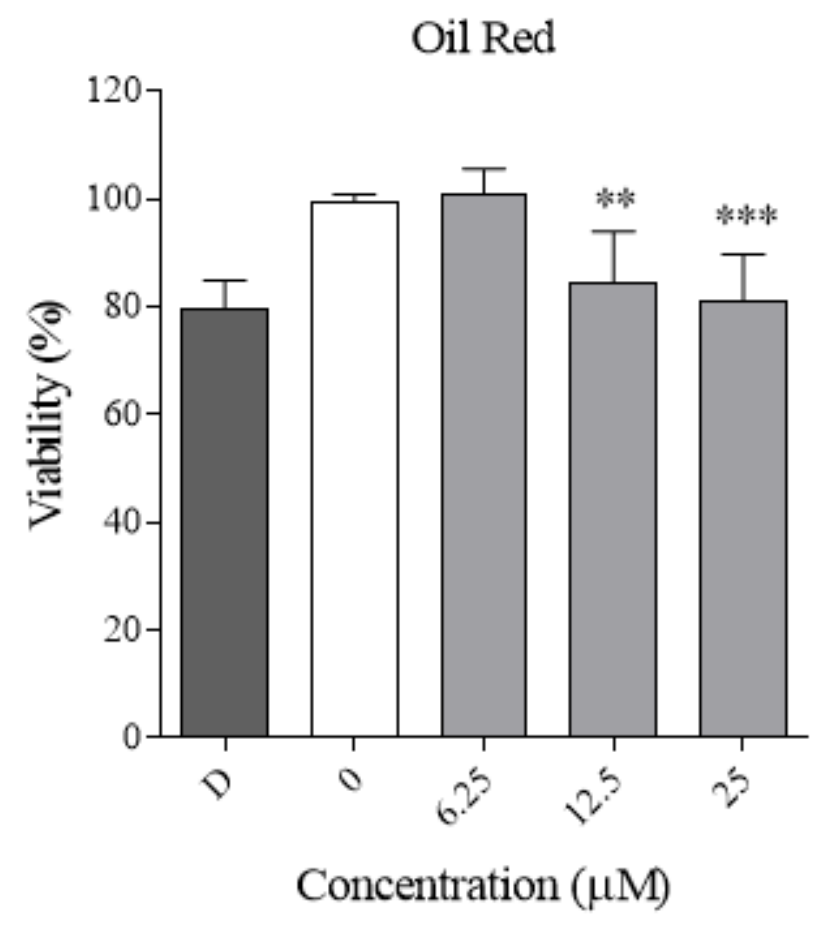

Figure 3

Effects of different concentrations of TQ on lipid differentiation in Oil Red 0 staining. Each test was repeated 3 times and represent as Mean \pm SD. $* \star=p<0.01$ and $* \star \star=p<0.001$ compared to the positive control 
Fig. 4

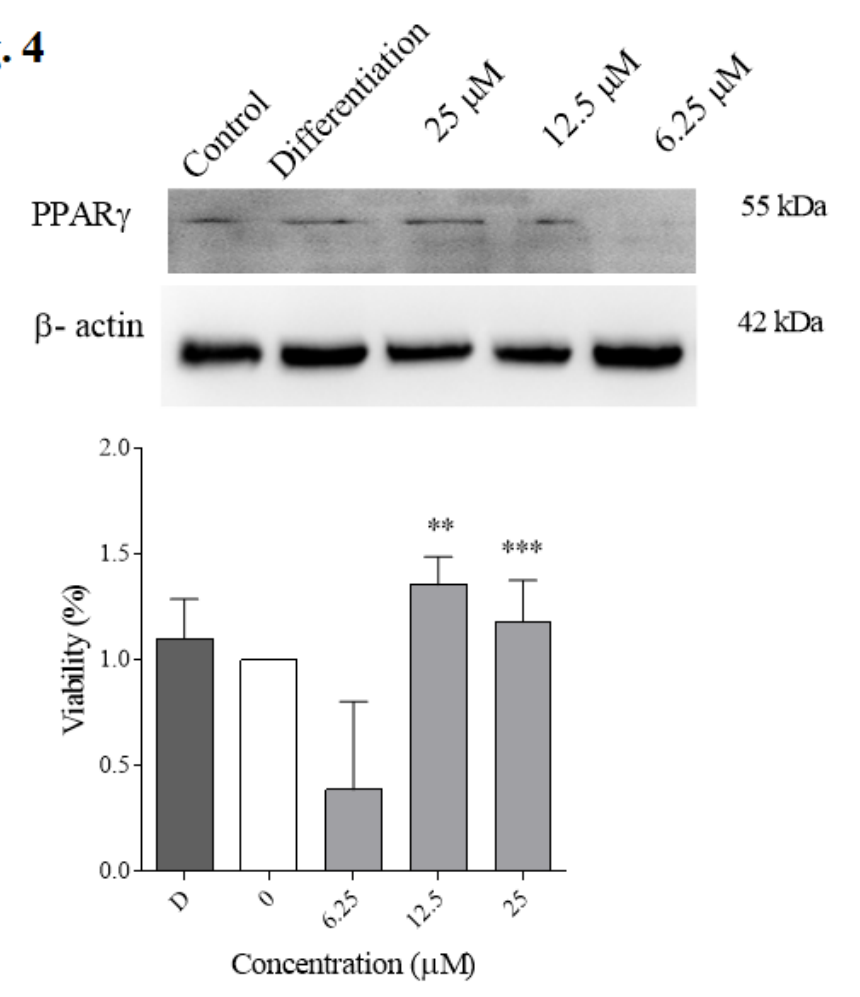

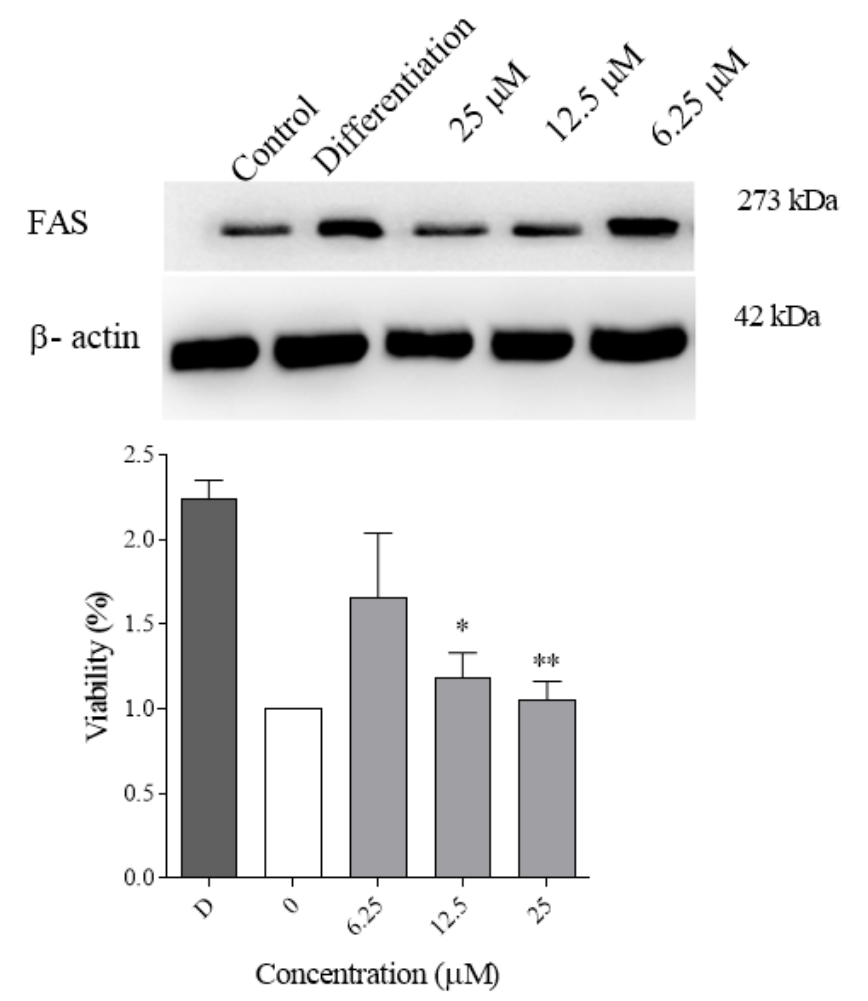

\section{Figure 4}

Western blot analysis of FAS, PPARy expression. Relative protein expression levels of FAS and PPARY in ADSCs in the different groups. Western blot analysis demonstrating the expression levels of the various proteins. $\beta$ actin used as a loading control. Results reported as Mean \pm SD. $*=p<0.05$ and $* *=p<0.01$ compared to the positive control (differentiation group). PPARY (Peroxisome proliferator-activated receptor Y); FAS (Fatty Acid Synthetase) 
Fig. 5

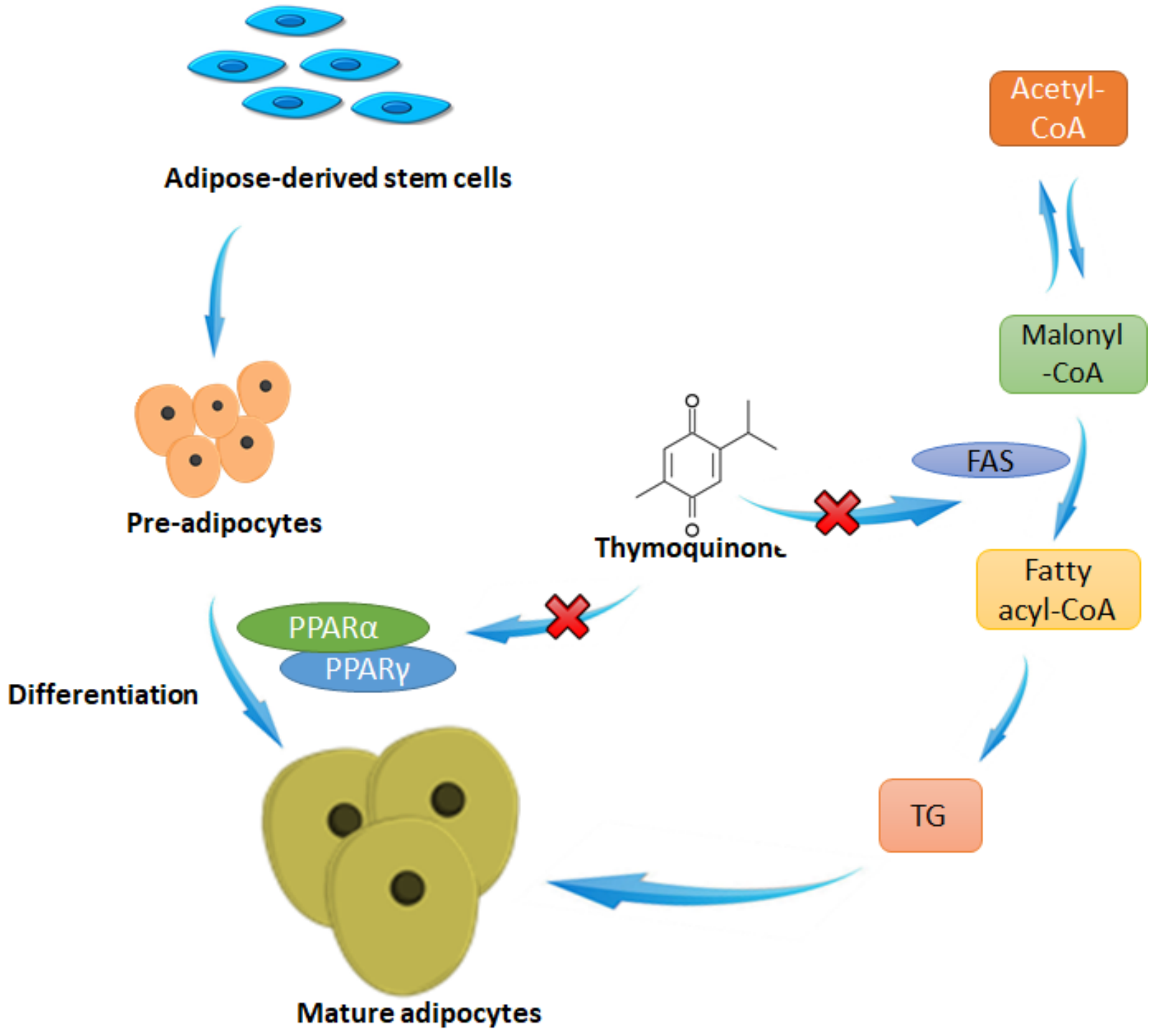

Figure 5

Molecular mechanisms of inhibition of adipocyte differentiation by TQ 